\title{
Down-regulation of Survivin by BIX-01294 Pretreatment Overcomes Resistance of Hepatocellular Carcinoma Cells to TRAIL
}

\author{
YENA NAMGUNG ${ }^{1}$, SO YOUNG KIM ${ }^{2}$ and INKI KIM ${ }^{1,3}$ \\ ${ }^{1}$ Department of Convergence Medicine, University of Ulsan College of Medicine, Seoul, Republic of Korea; \\ ${ }^{2}$ Biomedical Research Center, Asan Institute for Life Sciences, Asan Medical Center, Seoul, Republic of Korea; \\ ${ }^{3}$ Convergence Medicine Research Center, Asan Institute for Life Sciences, \\ Asan Medical Center, Seoul, Republic of Korea
}

\begin{abstract}
Background/Aim: Tumor necrosis factor-related apoptosis-inducing ligand (TRAIL) is a cancer-selective, cell-death-inducing agent with little toxicity to normal cells. However, various human cancers and cancer cell lines have been reported to be resistant to TRAIL. Molecular clarification of resistance mechanism is needed. Materials and Methods: Compound screening, proliferation assays, western blotting, and flow cytometry were used to examine the sensitizer activity of methyl transferase inhibitor BIX01294 in combination with TRAIL, in hepatocellular carcinoma (HCC) cells. RNA sequencing analysis and single guide (sg)RNA-mediated gene deletion were used to investigate the role of survivin in sensitization. Results: In HCC cells, BIX-01294 enhanced TRAIL sensitivity by reducing survivin expression at the RNA level. Small interference RNA-mediated gene knockdown demonstrated the mechanism of sensitization to be via the reduction of survivin. Conclusion: Euchromatin histone methyltransferase 2 (EHMT2) inhibition by BIX-01294 may be a potent antitumor therapeutic strategy for human HCC.
\end{abstract}

Hepatocellular carcinoma (HCC) is the most common cancer type originating from liver cells (1). HCC is thought to occur due to an imbalance between anti- and pro-apoptotic factors in liver cells $(2,3)$. Therefore, understanding the cellular mechanism regulating HCC cell apoptosis is useful for developing therapies for HCC.

Correspondence to: Inki Kim, Department of Convergence Medicine, University of Ulsan College of Medicine \& Asan Institute for Life Sciences, Asan Medical Center, 43gil Olympicro, Pungnapdong, Songpagu, Seoul, Republic of Korea. Tel: +82 230102515, e-mail: ik.kim@amc.seoul.kr

Key Words: TRAIL, hepatocellular carcinoma, EHMT2, survivin.
Tumor necrosis factor-related apoptosis-inducing ligand (TRAIL) is an inducer of apoptotic cell death with tumor cell selectivity, but has limited cytotoxicity to non-cancerous cells (4). Accordingly, the potential of TRAIL as an anticancer therapeutic agent has been examined (5-7). However, human cancers have exhibited both acquired and intrinsic resistance to TRAIL, which has led to failures in establishing clinically effective TRAIL-mediated therapies (8). Various reports have suggested that TRAIL resistance occurs in all the steps of the TRAIL-induced signaling pathway, from ligand-receptor interaction to the activation of executioner caspases $(8,9)$. Understanding the underlying molecular mechanisms of resistance to TRAIL in HCC using these sensitizers may be useful for HCC treatment.

Epigenetic regulation of proteins and histones, including methylation, plays various roles in a wide range of cell functions (10). Among the methylating enzymes, euchromatin histone methyltransferase 2 (EHMT2 or G9a) di-methylates H3 histone lysine residue 9 (11). The pro-tumorigenic role of EHMT2 is currently under investigation, as overexpression of EHMT2 has been reported in some types of cancers, including cancer of the bladder, lung, liver, and breast (12-14).

In the present study, we isolated BIX-01294, a chemical inhibitor of EHMT2, as a sensitizer of TRAIL-induced cell death in HCC cells and investigated the molecular mechanism of TRAIL sensitization by EHMT2 inhibition. Our results suggest that EHMT2 is a potent therapeutic target of HCC treatment.

\section{Materials and Methods}

Cell culture and reagents. Huh7, HepG2, and SNU475 HCC cells were from American Type Cell Culture (Manassas, VA, USA). All cells were cultured at $37^{\circ} \mathrm{C}$ in a humidified atmosphere containing $5 \% \mathrm{CO}_{2}$. Huh7 and HepG2 cells were cultured in Dulbecco's modified Eagle's medium containing 10\% fetal bovine serum, $2 \mathrm{mM}$ L-glutamine, and $100 \mathrm{U} / \mathrm{ml}$ penicillin/streptomycin. SNU475 cells 
were maintained in RPMI-1640 with the same supplements. BIX01294 was obtained from Sigma Aldrich (St. Louis, MO, USA).

TRAIL sensitizer screening. The TRAIL sensitizer screening procedure was performed as previously described (15). A collection of 80 cellular enzyme inhibitor compounds was tested. Untreated cells were used as control.

Western blot analysis. Cells were extracted in extraction buffer including phosphatase and protease inhibitors. Protein concentration was evaluated using a bicinchoninic acid assay kit (Pierce, Rockford, IL, USA). Cell extracts were then diluted in sample buffer, and $25 \mu \mathrm{g}$ cell extracts were used for western blotting. Protein samples, transferred to a polyvinyl difluoride membrane (Millipore, Billerica, MA), were blocked by 3\% to 5\% skim milk in Tris-buffered saline with TWEEN 20 (TBS-T). The membranes were incubated with the appropriate primary antibody (dilution $1: 1,000$ ) for more than $2 \mathrm{~h}$ at room temperature, or overnight at $4^{\circ} \mathrm{C}$, and were then washed with TBS-T three times. After the secondary antibody incubation (1:5,000 at room temperature), washes with TBS-T were repeated, and protein bands were visualized by an enhanced chemiluminescence detection kit.

The primary antibodies, anti-caspase- 8 (9746), anti-caspase-3 (9665), anti-poly-ADP-ribosyl polymerase (PARP) (9532), anti-BclxL (2764), anti-Mcl-1 (5453), anti-Bad (9292), anti-Bax (2772), antiBak (6947), anti-xIAP (2045), anti-Survivin (2808), anti-cIAP (7065), anti-EHMT2 (3306) were from Cell Signaling Technology (Danvers, MA, USA). Anti-PUMA (AB33906) antibody was purchased from Abcam (Cambridge, UK). Anti-tubulin-alpha antibody (Sigma Aldrich) and anti-beta-actin antibody (Novus) were used as loading control.

Cell proliferation assay and flow cytometry analysis. To evaluate cell proliferation, the CellTiterGlo assay (Promega, Madison, WI, USA) was conducted according to the manufacturer's instructions. Briefly, cells were seeded at a density of $3-4 \times 10^{3}$ cells/well in a 96well plate and pre-treated with BIX-01294 at the indicated concentrations for $1 \mathrm{~h}$, followed by treatment with TRAIL for an additional $24 \mathrm{~h}$. The luminescence value from each well was measured with a Victor3 label reader (PerkinElmer, Waltham, MA, USA). The raw value was transferred to Prism software (Graphpad, La Jolla, CA, USA) to evaluate relative cell viability.

For flow cytometry, cells were pelleted by centrifugation, washed with cold phosphate-buffered saline, and incubated with annexin $\mathrm{V}$ and propidium iodide $(50 \mu \mathrm{g} / \mathrm{ml})$ in assay buffer (Invitrogen, Carlsbad, CA, USA). After flow cytometry analysis by FACS Calibur (BD Biosciences, San Jose, CA, USA), the annexin V-positive proportion of the population was considered apoptotic.

Small interference (si)RNA transfection and viability assay. Survivin siRNA \#1 (5'-ACUUGGCCCAGUGUUUCUUCU-3') and siRNA \#2 (5'-GUGUCUGGACCUCAUGUUGUU-3') were synthesized by Bioneer (Daejeon, Korea). A control siRNA (5'-ACGUGACAC GUUCGGAGAAUU-3') was purchased from Dharmacon (Lafayette, CO, USA). In knockdown experiments, HCC cells were transfected with 40 pmol survivin siRNA with $8 \mu$ of RNAiMAX ${ }^{\mathrm{TM}}$ (Life Technologies, Carlsbad, CA, USA) for $48 \mathrm{~h}$.

RNA isolation, sample library preparation, and RNA sequencing. Total RNA was isolated from HCC cells (Huh7, HepG2, and SNU475) after incubation with or without BIX-01294. RNA quality was examined using an Agilent 2100 bioanalyzer with an RNA 6000 nano chip
Table I. List of genes that showed reduced expression (less than 0.7 fold) in hepatocellular carcinoma cells treated with $10 \mu \mathrm{M}$ BIX-01294, compared to control untreated cells.

\begin{tabular}{lccc}
\hline & \multicolumn{3}{c}{ Cell lines } \\
\cline { 2 - 4 } Gene & $\begin{array}{c}\text { HepG2 } \\
\text { (fold change) }\end{array}$ & $\begin{array}{c}\text { Huh7 } \\
\text { (fold change) }\end{array}$ & $\begin{array}{c}\text { SNU475 } \\
\text { (fold change) }\end{array}$ \\
\hline GJA9 & 0.452 & 0.289 & 0.363 \\
CCNB1 & 0.503 & 0.503 & 0.459 \\
BIRC5 & 0.449 & 0.504 & 0.476 \\
MYCBP & 0.442 & 0.290 & 0.351 \\
LINC00467 & 0.415 & 0.511 & 0.655 \\
ACTB & 0.558 & 0.650 & 0.593 \\
SPDL1 & 0.328 & 0.533 & 0.592 \\
\hline
\end{tabular}

(Agilent Technologies, Santa Clara, CA, USA). Construction of the RNA library was performed using the QuantSeq 3' mRNA-Seq Library Preparation Kit (Lexogen, Inc., Vienna, Austria). The product of the double-stranded nucleic acid library was isolated using magnetic beads to remove the reaction composites. The final library was purified from PCR components. High-throughput RNA sequencing was performed using the NextSeq 500 Sequencing System (Illumina, Inc., San Diego, CA, USA).

Generation of transgenic cell line. CRISPR cell lines were constructed using small guided (sg)RNA. The sequences for each EHMT2 deletion were: EHMT2 sgRNA\#1 - EHMT2-RG1F 5'-cacc AGAAGTGACCCTGACGAAAG-3' and EHMT2-RG1R 5'-aaac CTTTCGTCAGGGTCACTTCT-3', and EHMT2 sgRNA\#2 EHMT2-RG2F 5'-cacc CTACTATGATTCCTACTCTG-3' and EHMT2 RG2R 5'-aaac CAGAGTAGGAATCATAGTAG-3'.

After virus production by transfection, cells were infected by lentivirus particles, and clones were isolated in tissue culture media containing puromycin. The knockout efficiency of each clone was confirmed by western blotting.

Statistical analysis. All results are presented as mean \pm standard deviation (SD), and differences were identified using the $t$-test. Statistical significance of comparisons between each treatment groups was identified using one-way analysis of variance (ANOVA) test. All $p$-values $<0.05$ were considered statistically significant.

\section{Results}

BIX-01294 sensitizes HCC cells to TRAIL. The cell deathinducing activity of TRAIL was examined in eight HCC cell lines in a dose-dependent manner (Figure 1A). Even at $100 \mathrm{ng} / \mathrm{ml}$ TRAIL, which is an efficient concentration for inducing cell death in different cancer cell lines $(16,17)$, six of eight HCC cells showed less than $20 \%$ proliferation suppression in a cell proliferation assay, indicating that HCC cells were generally resistant to TRAIL. We next performed pilot scale compounds screening using cellular inhibitors from the library of pharmacologically active compounds (LOPAC; Sigma-Aldrich), to isolate chemical 
A

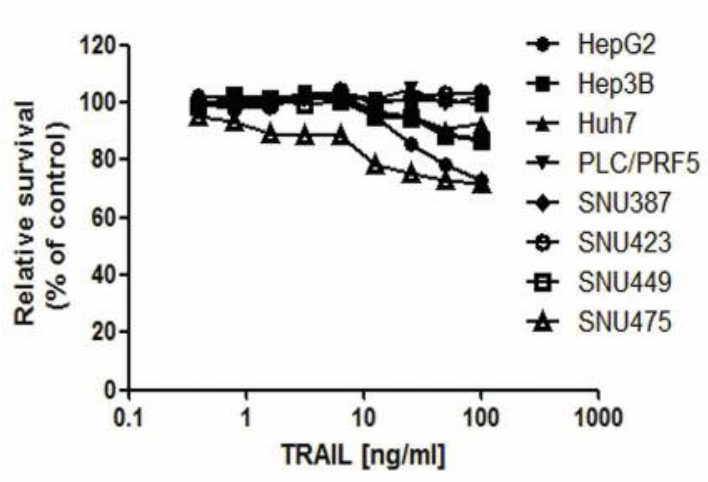

B

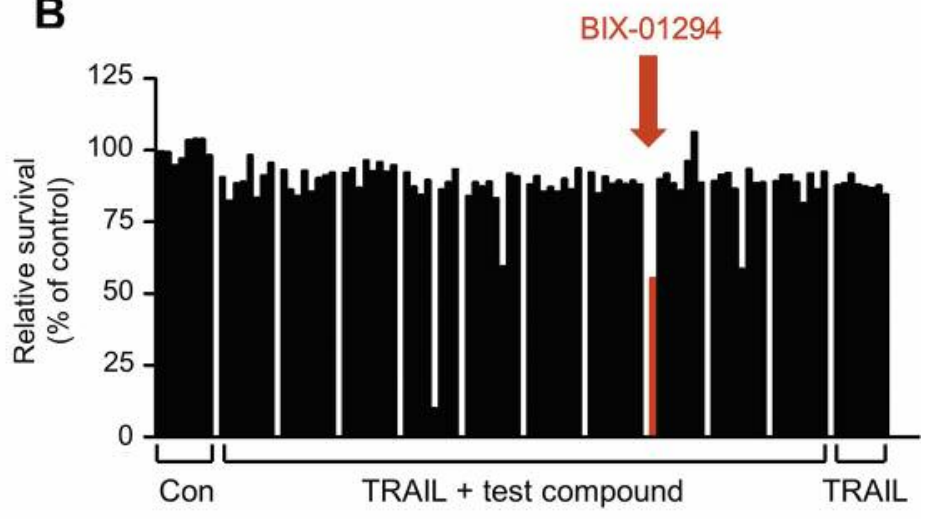

C

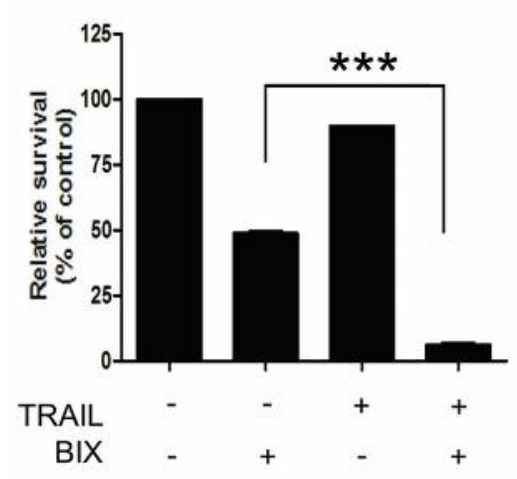

D

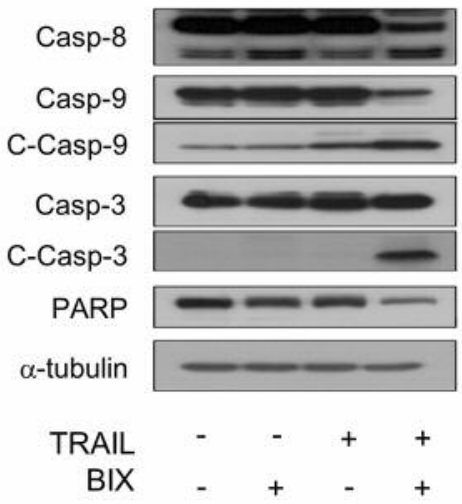

E

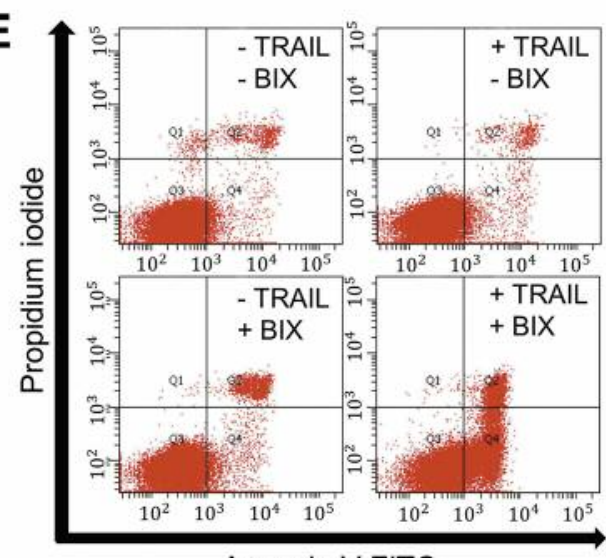

Annexin V-FITC

Figure 1. Resistance of liver cancer cells to TRAIL-induced cell death and small-molecule sensitizer screening. Hepatocellular carcinoma (HCC) cells were treated with TRAIL at increasing doses for $24 \mathrm{~h}$ and relative survival rates were evaluated by cell proliferation assay. Data represents mean \pm standard deviation (SD) from 2 independent experiments (A). A graph of the assay plate in which BIX-01294 was isolated as a TRAIL sensitizer is presented. The well with BIX-01294 is indicated with the red arrow (B). Relative survival of Huh7 cells exposed to TRAIL (200 ng/ml) with or without BIX-01294 (BIX; $10 \mu \mathrm{M})$ for $24 \mathrm{~h}$ was evaluated by proliferation assay. Data is presented as the mean $\pm S D$ of two independent experiments with triplicates (***p<0.001) (C). Apoptosis signaling in Huh7 cells treated with TRAIL (200 ng/ml) with or without BIX-01294 (10 $\mu M)$ were analyzed by western blotting. A representative result of two independent experiments is shown (D). Apoptotic activity in Huh7 cells exposed to TRAIL (200 $\mathrm{ng} / \mathrm{ml})$ with BIX-01294 (10 $\mu \mathrm{M})$ was evaluated. Incubated cells were stained with annexin $V$ with propidium iodide and analyzed by flow cytometry. Representative assay images are presented from two independent experiments (E). Con, Control untreated cells; Casp, caspase; C-Casp, cleaved caspase.

sensitizers of TRAIL. Each well was pre-treated with the library compounds $(5 \mu \mathrm{M})$ for $1 \mathrm{~h}$, and cells were exposed to a non-lethal dose of recombinant human TRAIL $(100 \mathrm{ng} / \mathrm{ml})$ for $24 \mathrm{~h}$. On the second day, cell survival was evaluated.

In the primary screen, the BIX-01294 showed a marginal level of sensitization in Huh7 cells (Figure 1B) and was selected as a candidate TRAIL sensitizer. In the next step, the sensitizer activity of BIX-01294 was again confirmed via the same assay as in primary screening (Figure 1C). Since the viability index in Figure 1B does not reflect single treatment of TRAIL or BIX-01294, we compared the relative viability following combination treatment with that following single treatment after a longer incubation period.
As shown in Figure 1C, BIX-01294 single treatment decreased cell proliferation to $55 \%$, while combination treatment of TRAIL with BIX-01294 strongly decreased cell proliferation to $25 \%$.

To confirm that proliferation inhibition by combination treatment was associated with apoptosis induction, we examined apoptosis by western blotting and flow cytometry analysis. Figure 1D shows the activation of caspases and a decrease in caspase substrate poly-ADP-ribose polymerase (PARP). Combination treatment increased the annexin V-positive population to $37 \%$, while the effects of TRAIL (7\%) and BIX-01294 single treatment (11\%) were marginal (Figure 1E). 
A

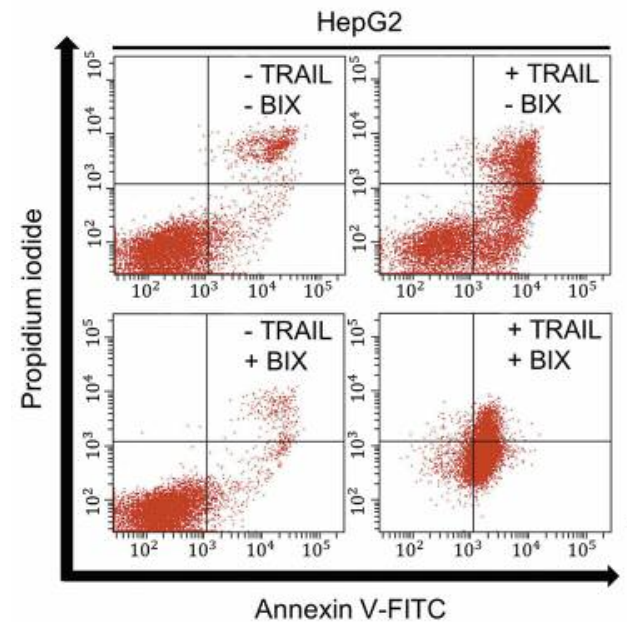

C

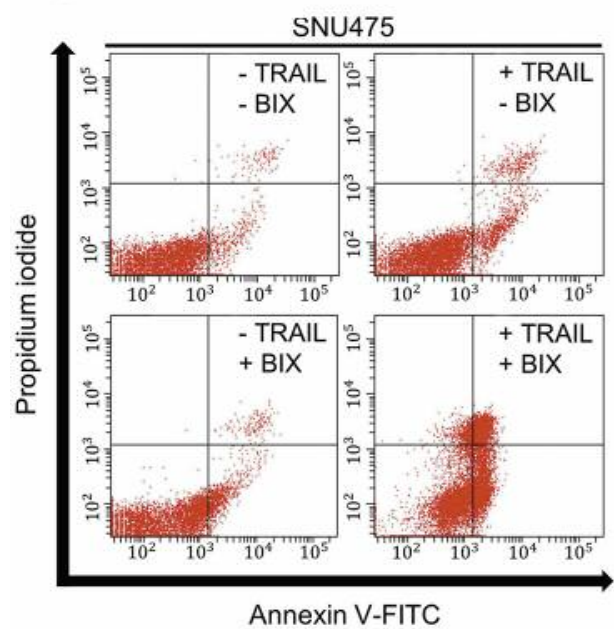

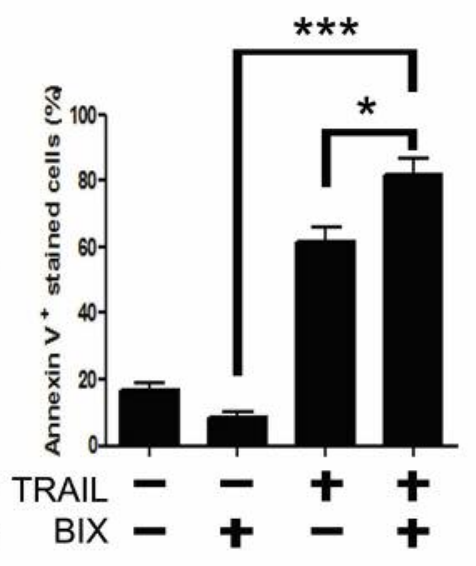

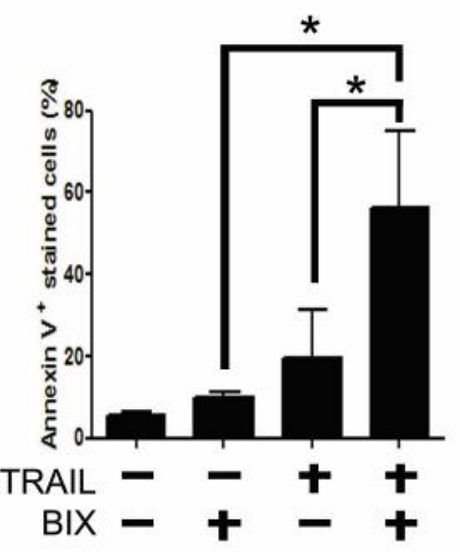

B

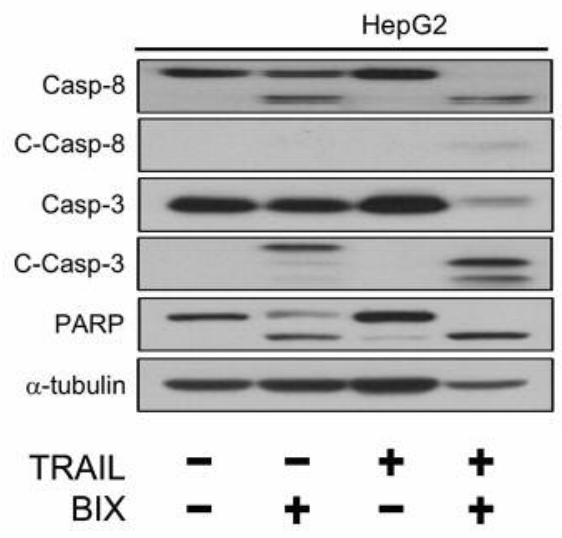

D

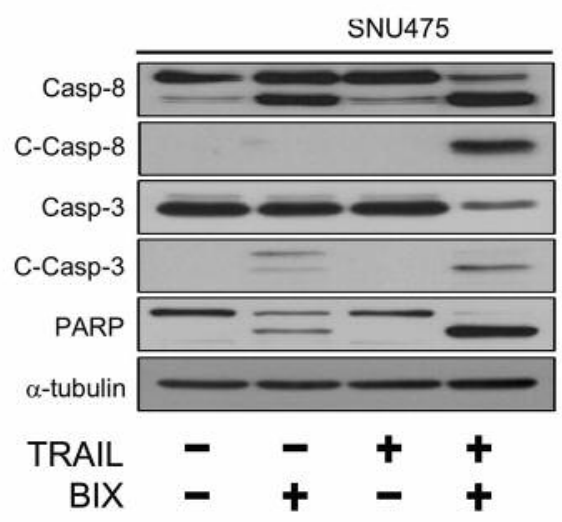

Figure 2. BIX-01294 sensitized various hepatocellular carcinoma (HCC) cell lines to TRAIL-induced apoptosis. Apoptotic activities in HepG2 cells (A) and SNU475 cells $(C)$ after exposure to TRAIL with or without BIX-01294 were analyzed via flow cytometry. Representative assay images from two independent experiments are presented (left panel). The percentage of apoptotic annexin $V$-stained cells were presented as a graph at right panel $(n=2$, mean \pm standard deviation, $* p<0.05$, ***p<0.001). The expression of apoptotic markers in HepG 2 cells $(B)$ and SNU475 cells $(D)$ treated with TRAIL and/or BIX-01294 was analyzed by western blotting. Representative assay images from two independent experiments are presented. Casp, Caspase; C-Casp, cleaved caspase.

BIX-01294 sensitizes various HCC cells to TRAIL. To examine the cell type specificity of BIX-01294 as a TRAIL sensitizer, two additional HCC cell lines were tested with TRAIL and BIX-01294 alone, or both.

As shown in Figure 2, BIX-01294 sensitized HepG2 and SNU 475 cells to TRAIL, revealed via western blotting, and flow cytometry analyses.

BIX-01294 down-regulates survivin in HCC cells. To understand the underlying mechanism of sensitization, we explored the changes in apoptosis-related protein expression. As shown in Figure 3A, combination treatment with BIX-01294 and TRAIL significantly reduced anti-apoptotic protein expression. From the tested proteins, only survivin was down-regulated by single treatment with BIX-01294. We next examined whether survivin was similarly down-regulated by BIX-01294 in other cells. As shown in Figure 3B and C, the expression of survivin was strongly down-regulated by BIX-01294 with or without TRAIL in HepG2 and SNU475 cells, indicating that survivin is a common target regulated by BIX-01294. 
A

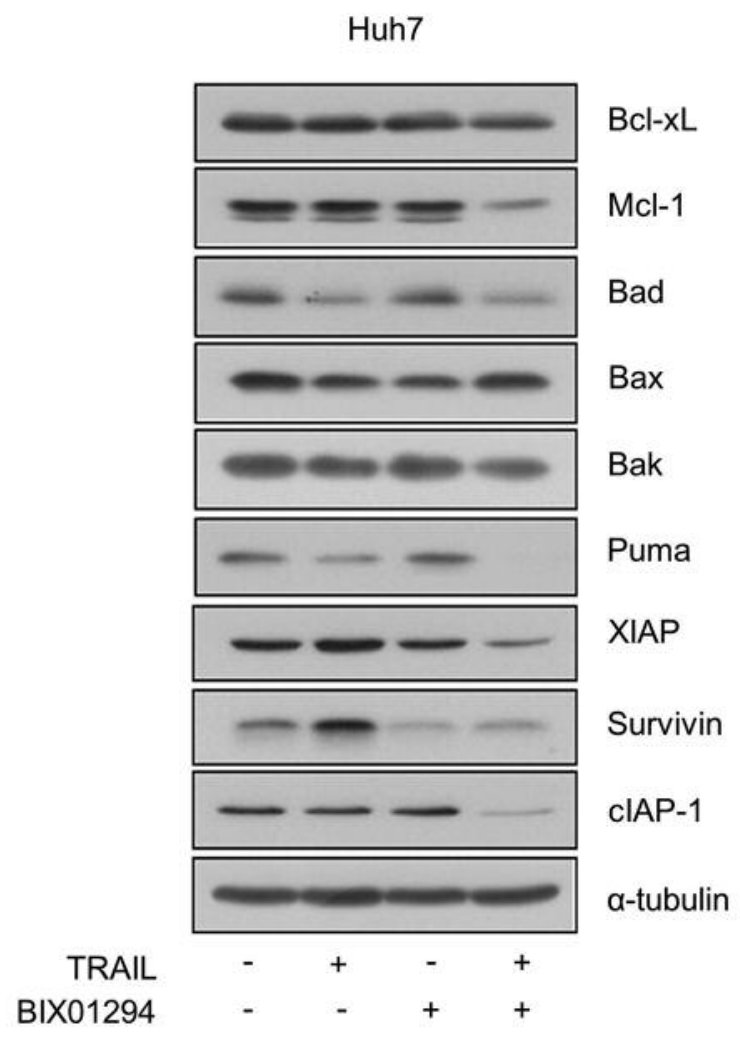

B

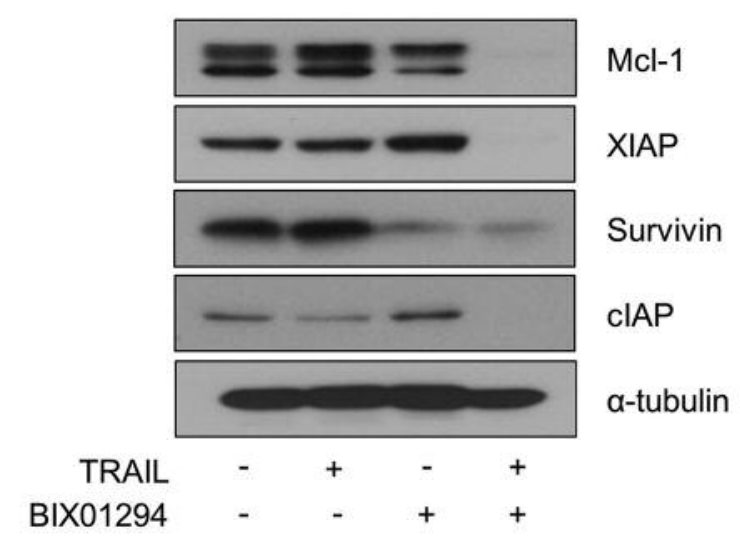

C

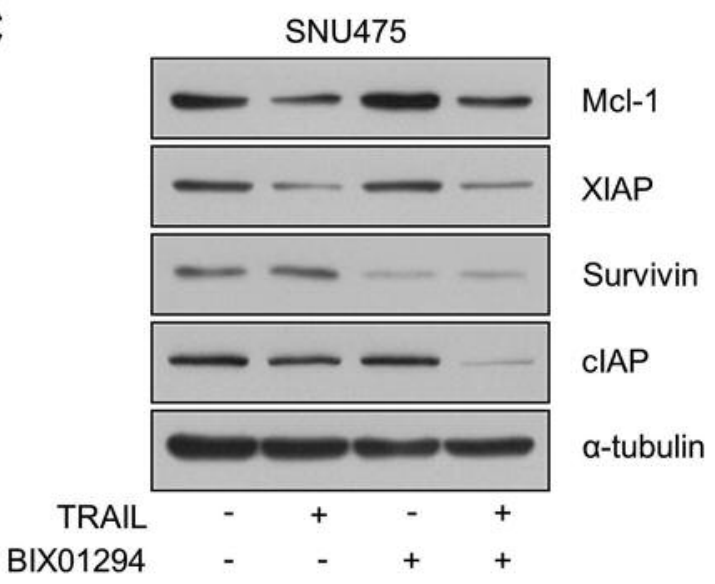

D

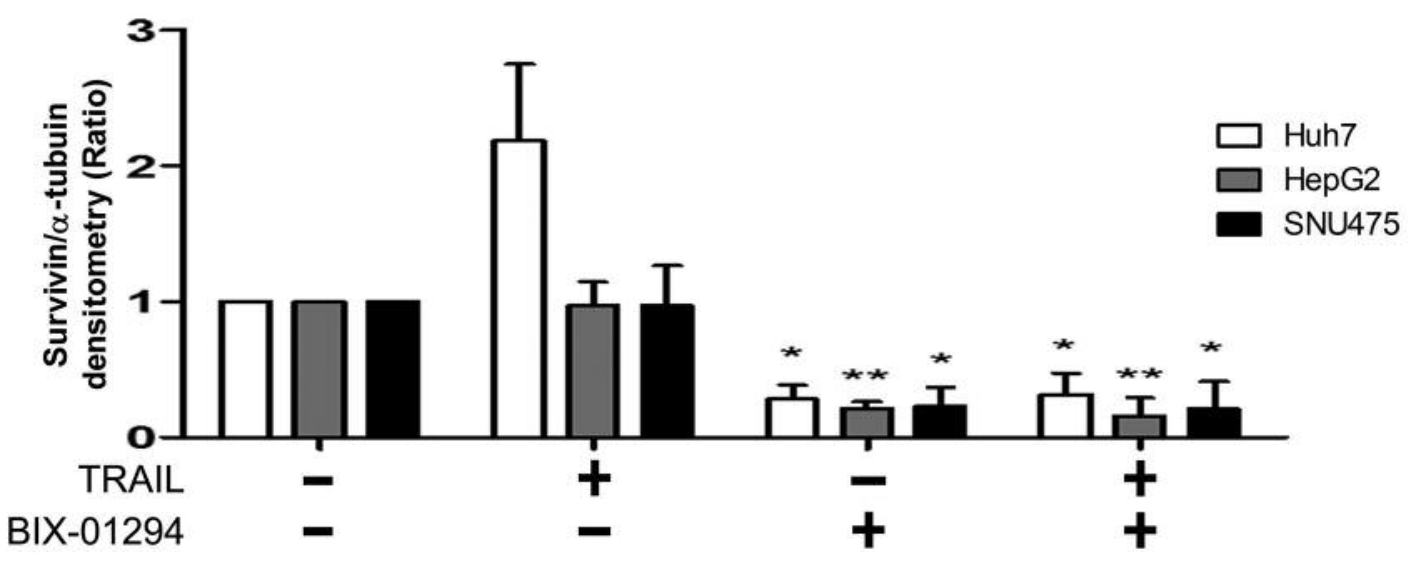

Figure 3. Survivin was down-regulated by BIX-01294. Cell extracts of Huh7 (A), HepG2 (B), and SNU475 cells (C) exposed to TRAIL with or without BIX-01294 were analyzed for the expression of apoptosis-related proteins. Representative assay images are presented from two independent experiments. The fold ratio of survivin band intensity by each treatment was normalized to tubulin bands and presented $(n=2, * p<0.05$, **p $<0.005$ compared to control) $(D)$.

Down-regulation of survivin is the underlying mechanism of TRAIL sensitization by BIX-01294. The molecular mechanism of survivin down-regulation by BIX-01294 was analyzed via RNA sequencing analysis. The Venn diagram in Figure 4A shows the number of down-regulated genes following incubation of HCC cells with $10 \mu \mathrm{M}$ BIX-01294. A total of 14 genes were commonly down-regulated by BIX01294 treatment in all tested HCC cells. Among these, seven 
A
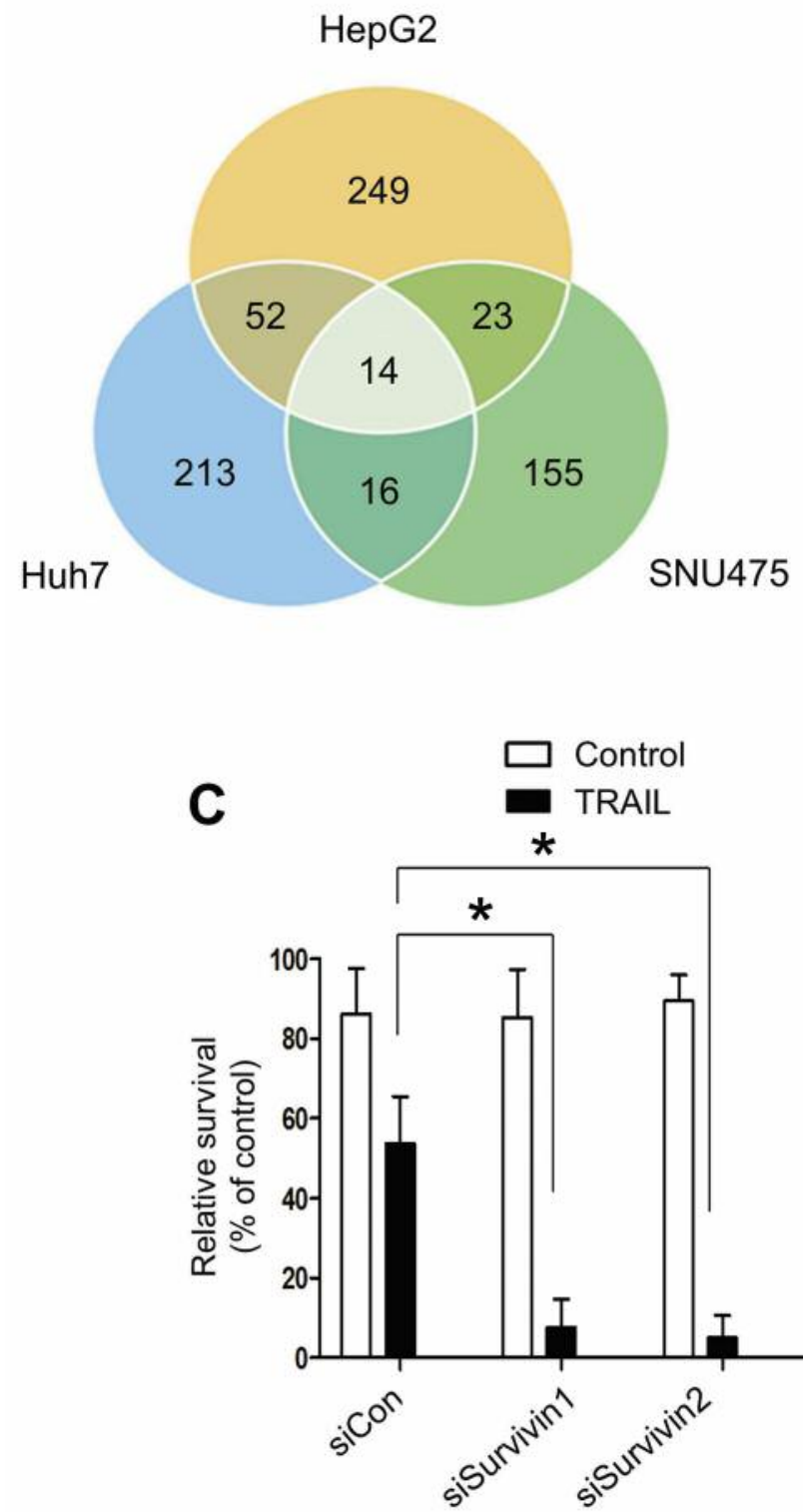

B

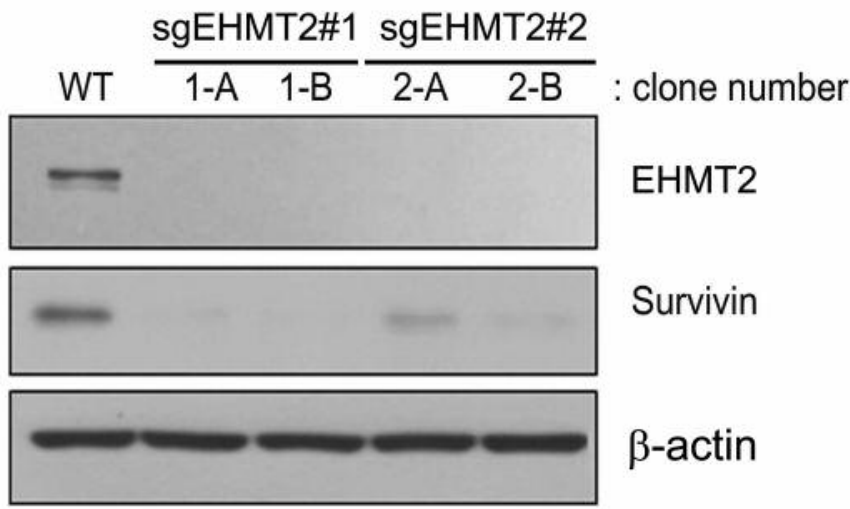

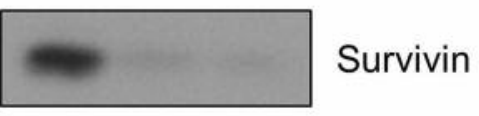

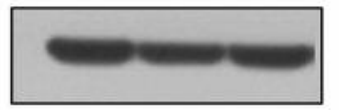

a-tubulin

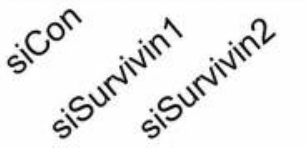

Figure 4. EHMT2-mediated survivin expression regulates the sensitivity of HCC cells to TRAIL. The Venn diagram shows the number of downregulated genes by BIX-01294 in each cell line (A). EHMT2-I- SNU475 cells were selected, and the expression of survivin was analyzed via western blotting. Representative assay images are presented from two independent experiments $(B)$. HepG2 cells were transfected with control siRNA or survivin siRNA (40 pmol) and treated with TRAIL. Cell viability was evaluated by trypan blue exclusion. Data represent mean \pm standard deviation $(n=2, * p<0.05)$. The knockdown efficiency of survivin siRNA was confirmed by western blotting $(C)$.

genes showed a fold change of less than 0.7 , including survivin (BIRC5) (Table I).

The role of EHMT2 in survivin down-regulation was confirmed by western blotting in EHMT2 knockout cells. We constructed EHMT2 knockout models in SNU475 cells using a CRISPR system with two kinds of sgRNA oligos. As shown in Figure 4B, knockout of the EHMT2 gene down- regulated survivin expression, a result that concurs with RNA sequencing data.

We next examined whether survivin down-regulation is the main cause of sensitization by BIX-01294 to TRAIL. Two (siRNAs for survivin were used to examine the role of survivin down-regulation in TRAIL-induced apoptosis. Transfection of HCC cells with the two siRNAs strongly 
reduced survivin expression, which sensitized HepG2 cells to TRAIL (Figure 4C).

\section{Discussion}

In this study, BIX-01294, a small molecule inhibitor of EHMT2 (G9a), was identified as a TRAIL sensitizer in HCC cells. Since the sensitizer function of BIX-01294 was observed in three different HCC cell lines, the underlying molecular mechanism of BIX-01294 in sensitizing cells to TRAIL may be a promising HCC treatment strategy. Moreover, our results supported that the anti-apoptotic protein survivin can be regulated by BIX01294, and additionally, it plays a key role in BIX-01294induced TRAIL sensitization in HCC cells (Figure 4).

Survivin is a member of the inhibitor of apoptosis protein (IAP) family; it prevents apoptotic cell death by inhibiting caspase activation (18), and its expression is highly correlated with tumor characteristics (18). The expression of survivin has been reported to be regulated at the transcriptional and post-translational levels (19-21). Of the various mechanisms regulating survivin expression, ubiquitin-proteasome-dependent degradation is of interest because the expression of survivin changes temporarily depending on the phase of the cell cycle (20). However, our data showed that BIX-01294 down-regulated survivin mRNA at the transcriptional level, indicating that various mechanisms at the transcription and post-transcription levels are involved in survivin expression.

EHMT2 is an epigenetic enzyme responsible for histone 3 lysine 9 dimethylation (H3K9me) (22). H3K9me is a tag for epigenetic transcriptional repression, while it also recruits heterochromatin protein 1 (HP1), which induces compaction of chromatin (22). EHMT2 is considered to play a crucial role in gene silencing and gene expression-related cellular responses to various conditions, including cell death and autophagy.

In our study, inhibition of EHMT2 led to the downregulation of survivin mRNA, suggesting that regulation of the anti-apoptotic program via survivin is related to EHMT2. Although a wide range of cell functions are associated with EHMT2, the relationship between EHMT2 and survivin remains unclear. Studies are needed to clarify the mechanism of survivin down-regulation via EHMT2.

\section{Authors' Contributions}

YNNG: Designed and performed experiments; SYK: Designed and performed experiments, prepared manuscript; IKK: Designed experiments, analyzed data, prepared manuscript.

\section{Acknowledgements}

This study was supported by the Basic Science Research Program (50\%) (NRF-2016R1D1A1B03932365) funded by the Ministry of Science, Information \& Communication Technology (ICT) and
Future Planning; a grant (40\%) from the Yujeonja-Donguibogam project (NRF-2017M3A9C4065958), under the Ministry of Science, Information \& Communication Technology (ICT) and Future Planning; and an internal research grant (10\%) from the Asan Institute for Life Sciences (2017-512). We thank the core facility of the ConveRgence mEDIcine research cenTer (CREDIT) for support and instrumentation.

\section{References}

1 Yang JD and Roberts LR: Hepatocellular carcinoma: A global view. Nat Rev Gastroenterol Hepatol 7(8): 448-458, 2010. PMID: 20628345. DOI: $10.1038 /$ nrgastro.2010.100

2 Fabregat I: Dysregulation of apoptosis in hepatocellular carcinoma cells. World J Gastroenterol 15(5): 513-520, 2009. PMID: 19195051.

3 HM EI-E, Saad EA, Toson EA, Abdel Malak CA and Gadelhak NA: Apoptosis and cell proliferation: Correlation with bcl-2 and p53 oncoprotein expression in human hepatocellular carcinoma. Hepatogastroenterology 61(133): 1393-1401, 2014. PMID: 25436317.

4 Walczak H, Miller RE, Ariail K, Gliniak B, Griffith TS, Kubin M, Chin W, Jones J, Woodward A, Le T, Smith C, Smolak P, Goodwin RG, Rauch CT, Schuh JC and Lynch DH: Tumoricidal activity of tumor necrosis factor-related apoptosis-inducing ligand in vivo. Nat Med 5(2): 157-163, 1999. PMID: 9930862. DOI: $10.1038 / 5517$

5 Newsom-Davis T, Prieske S and Walczak H: Is trail the holy grail of cancer therapy? Apoptosis 14(4): 607-623, 2009. PMID: 19194800. DOI: 10.1007/s10495-009-0321-2

6 Rahman M, Pumphrey JG and Lipkowitz S: The trail to targeted therapy of breast cancer. Adv Cancer Res 103: 43-73, 2009. PMID: 19854352. DOI: 10.1016/S0065-230X(09)03003-6

7 Stuckey DW and Shah K: Trail on trial: Preclinical advances in cancer therapy. Trends Mol Med 19(11): 685-694, 2013. PMID: 24076237. DOI: 10.1016/j.molmed.2013.08.007

8 Dimberg LY, Anderson CK, Camidge R, Behbakht K, Thorburn A and Ford HL: On the trail to successful cancer therapy? Predicting and counteracting resistance against trail-based therapeutics. Oncogene 32(11): 1341-1350, 2013. PMID: 22580613. DOI: $10.1038 /$ onc.2012.164

9 Johnstone RW, Ruefli AA and Lowe SW: Apoptosis: A link between cancer genetics and chemotherapy. Cell 108(2): 153164, 2002. PMID: 11832206.

10 Huang $\mathrm{J}$ and Berger SL: The emerging field of dynamic lysine methylation of non-histone proteins. Curr Opin Genet Dev 18(2): 152-158, 2008. PMID: 18339539. DOI: 10.1016/j.gde. 2008.01.012

11 Tachibana M, Sugimoto K, Fukushima T and Shinkai Y: Set domain-containing protein, $\mathrm{a}$, is a novel lysine-preferring mammalian histone methyltransferase with hyperactivity and specific selectivity to lysines 9 and 27 of histone H3. J Biol Chem 276(27): 25309-25317, 2001. PMID: 11316813. DOI: 10.1074/jbc.M101914200

12 Cho HS, Kelly JD, Hayami S, Toyokawa G, Takawa M, Yoshimatsu M, Tsunoda T, Field HI, Neal DE, Ponder BA, Nakamura Y and Hamamoto R: Enhanced expression of ehmt2 is involved in the proliferation of cancer cells through negative regulation of siah1. Neoplasia 13(8): 676-684, 2011. PMID: 21847359 . 
13 Yokoyama M, Chiba T, Zen Y, Oshima M, Kusakabe Y, Noguchi Y, Yuki K, Koide S, Tara S, Saraya A, Aoyama K, Mimura N, Miyagi S, Inoue M, Wakamatsu T, Saito T, Ogasawara S, Suzuki E, Ooka Y, Tawada A, Otsuka M, Miyazaki M, Yokosuka O and Iwama A: Histone lysine methyltransferase g9a is a novel epigenetic target for the treatment of hepatocellular carcinoma. Oncotarget 8(13): 21315-21326, 2017. PMID: 28423509. DOI: 10.18632/oncotarget.15528

14 Kim K, Son MY, Jung CR, Kim DS and Cho HS: Ehmt2 is a metastasis regulator in breast cancer. Biochem Biophys Res Commun 496(2): 758-762, 2018. PMID: 29337058. DOI: 10.1016/j.bbrc.2018.01.074

15 Oh B, Park S, Pak JH and Kim I: Downregulation of MCL-1 by daunorubicin pretreatment reverses resistance of breast cancer cells to tnf-related apoptosis-inducing ligand. Biochem Biophys Res Commun 422(1): 42-47, 2012. PMID: 22554523. DOI: 10.1016/j.bbrc.2012.04.093

16 Zhang N, Wang X, Huo Q, Li X, Wang H, Schneider P, Hu G and Yang Q: The oncogene metadherin modulates the apoptotic pathway based on the tumor necrosis factor superfamily member trail (tumor necrosis factor-related apoptosis-inducing ligand) in breast cancer. J Biol Chem 288(13): 9396-9407, 2013. PMID: 23408429. DOI: $10.1074 /$ jbc.M112.395913

17 Rahman M, Davis SR, Pumphrey JG, Bao J, Nau MM, Meltzer PS and Lipkowitz S: Trail induces apoptosis in triple-negative breast cancer cells with a mesenchymal phenotype. Breast Cancer Res Treat 113(2): 217-230, 2009. PMID: 18266105. DOI: $10.1007 / \mathrm{s} 10549-008-9924-5$
18 Altieri DC: Survivin, cancer networks and pathway-directed drug discovery. Nat Rev Cancer 8(1): 61-70, 2008. PMID: 18075512. DOI: $10.1038 /$ nrc2293

19 Hoffman WH, Biade S, Zilfou JT, Chen J and Murphy M: Transcriptional repression of the anti-apoptotic survivin gene by wild type p53. J Biol Chem 277(5): 3247-3257, 2002. PMID: 11714700. DOI: $10.1074 /$ jbc.M106643200

20 Zhao J, Tenev T, Martins LM, Downward J and Lemoine NR: The ubiquitin-proteasome pathway regulates survivin degradation in a cell cycle-dependent manner. J Cell Sci $113 \mathrm{Pt}$ 23: 4363-4371, 2000. PMID: 11069780.

21 Tecleab A and Sebti SM: Depletion of k-ras promotes proteasome degradation of survivin. Cell Cycle 12(3): 522-532, 2013. PMID: 23324341. DOI: 10.4161/cc.23407

22 Sharma S, Kelly TK and Jones PA: Epigenetics in cancer. Carcinogenesis 31(1): 27-36, 2010. PMID: 19752007. DOI: 10.1093/carcin/bgp220 